\title{
Simulation analysis of fin swimming with bi-fins
}

\author{
Motomu NAKASHIMA*, Takuro YONEDA* ${ }^{*}$ and Tetsuro TANIGAWA ${ }^{* *}$ \\ *School of Engineering, Tokyo Institute of Technology \\ 2-12-2 Ookayama, Meguro-ku, Tokyo 152-8552, Japan \\ E-mail: motomu@sc.e.titech.ac.jp \\ ${ }^{\star *}$ Faculty of Human Sciences, Osaka International University
}

Received: 3 January 2019; Revised: 6 June 2019; Accepted: 1 July 2019

\begin{abstract}
The objective of this study was to simulate the swimming motion with bi-fins and investigate the effect of ankle joint motion by simulation. A model of a swimmer was constructed by utilizing the swimming human simulation model SWUM. The bi-fin was modeled as a series of five rigid plates. One rigid plate was connected to another by virtual springs and dampers. In order to determine the spring constants of the springs and damping coefficients of the dampers, static and dynamic bending tests were conducted. In order to determine the fluid force coefficients for the fin, an experiment in which the fin was located vertically in a pool and was moved horizontally by an air cylinder was conducted. In order to acquire the motion of swimming with fins, two swimmers were asked to perform maximal front crawl swimming with/without fins in an indoor swimming pool. The swimming motion was filmed by three cameras underwater and one camera on land. Using all of the input data, simulations reproducing the experimental trials were carried out. By the simulation, the developed simulation method was validated since the simulated swimming speeds were consistent with the experimental values in errors less than $6 \%$ of accuracy. By analyses with the validated simulation method, it was found that the smaller ankle joint movements resulted in a faster swimming speed. It was also found that the fixed angle of ankle joints affected the body pitch angle and that there is an optimum fixed angle for a swimmer to maximize swimming speed.
\end{abstract}

Keywords : Swimming, Bi-fin, Simulation, Sports biomechanics, Sports engineering

\section{Introduction}

Fin swimming is a sport in which swimmers attach fins and compete based on speed at the water surface or below the water surface. The first European championship for fin swimming was held in 1967, and the first world championship was held in 1976. In fin swimming races, two kinds of fins are used, a monofin worn by a swimmer with both feet attached to a single fin and a bi-fin worn by a swimmer with one on each foot. The bi-fin events in competition have a short history. Only the surface event, in which a swimmer swims at the water surface in the crawl stroke, has been adopted since the world championship in 2007.

There are few studies about swimming with bi-fins. For example, Zamparo et al. (2002 and 2006) investigated how fins affected the economy and efficiency on swimming. Nicolas et al. (2010) developed a new system for evaluation of fins in which a fin was mechanically driven in a human-like motion. Ongun (2014) investigated the effects of hand paddles and fins on speed and stroke frequency in freestyle swimming. However, the swimming motion itself in swimming with bi-fins has not been discussed in those previous studies. In particular, the motion and role of the ankle joints are expected to be different from those in swimming without fins, since the foot is virtually enlarged by attaching a large flexible fin. To investigate the effect of the motion of the ankle joints, simulation is an effective method. Although there are a few simulation studies about monofin swimming (Luersen et al., 2006; Bideau et al., 2010; Nakashima et al., 2010b), there has not been a simulation study for bi-fin swimming.

The objective of this study was to simulate the swimming motion with bi-fins and investigate the effect of ankle joint motion by simulation. 


\section{Simulation Method}

\subsection{Overview}

In the simulation method of the present study, the model of a swimmer with bi-fins consisted of the model of a swimmer and the model of bi-fins, as shown in Fig. 1(a). The model of a swimmer was constructed by utilizing the swimming human simulation model SWUM (Nakashima et al., 2007). SWUM was designed to solve the six degrees-offreedom absolute movement of the whole swimmer's body as a single rigid body with time integration using the input data of the swimmer's body geometry and relative joint motion. As shown in Fig. 1(b), the swimmer's body is represented by a series of 21 rigid body segments, which are as follows: lower waist, upper waist, lower chest, upper chest, shoulders, neck, head, upper hip, lower hip, thighs (right and left), shanks (right and left), feet (right and left), upper arms (right and left), forearms (right and left) and hands (right and left). Each body segment is represented by a truncated elliptic cone. The unsteady fluid force and gravitational force are taken into account as external forces acting on the whole body. The unsteady fluid force is assumed to be the sum of the inertial force due to the added mass of the fluid, normal and tangential drag forces and buoyancy. Without solving flow, these components are assumed to be computable from the local position, velocity, acceleration, direction, angular velocity, and angular acceleration for each part of the human body at each time step. Many other studies using SWUM, including its validation and application, have already been conducted to date (Nakashima, 2007; Nakashima, 2009; Kiuchi et al., 2010; Nakashima, 2010; Nakashima et al., 2010a; Nakashima et al., 2010b; Nakashima et al., 2012; Nakashima et al., 2013; Nakashima and Ono, 2014; Nakashima et al., 2018).

The bi-fin was modeled as a series of five rigid plates. One rigid plate was connected to another by virtual springs and dampers. The first set of spring and damper was for translational connection between the edges of two adjacent rigid plates, as shown in Fig. 1(c). The spring and damping coefficients had to be sufficiently large since the two edges of the adjacent rigid plate should not have relative displacement. The second set of spring and damper was for rotational displacement between two adjacent rigid plates, as shown in Fig. 1(d). The spring and damping coefficients corresponded to the actual rigidity (bending stiffness) of the fin. Therefore, these coefficients were determined based on experiments, as described in the next section.

In total, there were eleven rigid bodies in the present simulation, that is, the swimmer (one rigid body) and the two fins (five rigid bodies for each fin). The equations of motions of these eleven rigid bodies were simultaneously solved

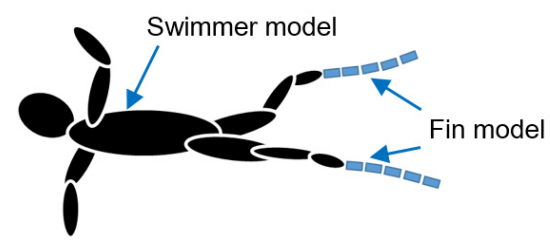

(a)

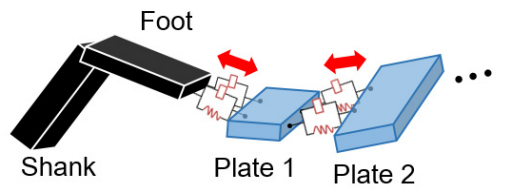

(c)

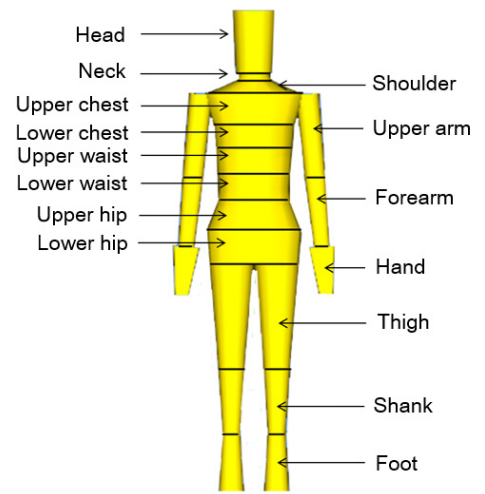

(b)

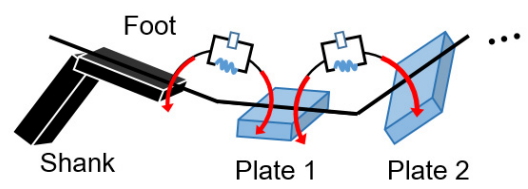

(d)

Fig. 1 The simulation models used in the present study. (a) Overview of swimmer model and fin model as series of five rigid plates, (b) swimmer model consisting of 21 body segments, (c) schematic view of translational connections between the swimmer's body and rigid plates, (d) schematic view of rotational connections between the swimmer's body and rigid plates. 
using the Runge-Kutta method, considering the external forces (fluid forces including buoyancy, gravity) as well as interaction among the rigid bodies. Such a calculation was enabled in the previous study as "multi agent/object simulation" (Nakashima et al., 2010a). The implementation in the present study is the same as was used in the previous study.

\subsection{Fin model}

The detailed geometry of the fin model is shown in Fig. 2a. The target fin in the present study was the NAJADE SPRINT fin, which is popular among bi-fin swimmers. In the model, the foot pocket part was reproduced by modification of the geometry of the foot of the swimmer. The blade part was reproduced with five rigid plates. The geometry of each part was measured and put into the model. Since the fin was almost neutrally buoyant, the density of the foot pocket part was given as the same as the swimmer (1.042), and that of the blade part was simply given as 1.0.

The spring constants and damping coefficients for the virtual rotational springs and dampers between the five rigid plates were determined. For the spring constants, a static bending test was conducted. In this test, six markers were attached on the side edge of a fin, and a load was applied by hanging a weight at the end edge of the fin, as shown in Fig. 3(a). The displacements of the markers were measured. The mass of the weight was changed from $0 \mathrm{~kg}$ to $5 \mathrm{~kg}$ with increments of $1 \mathrm{~kg}$. In order to obtain the spring coefficients in the model for the down kick as well as up kick, the fin was bent by the weight in both directions. On the other hand, a simulation reproducing the experimental situation of the static bending test was carried out, as shown in Fig. 2(b). The spring constants were determined so that the displacements of the markers in the experiment and simulation were consistent with each other as much as possible. An example of the results are shown in Fig. 3(b). This is the case of a down kick when the mass of the weight was $5 \mathrm{~kg}$.

For the damping coefficients, a dynamic bending test was conducted. The same experimental setup was used for this test. However, in the dynamic test, the string between the fin and the weight was cut with scissors so that the fin was

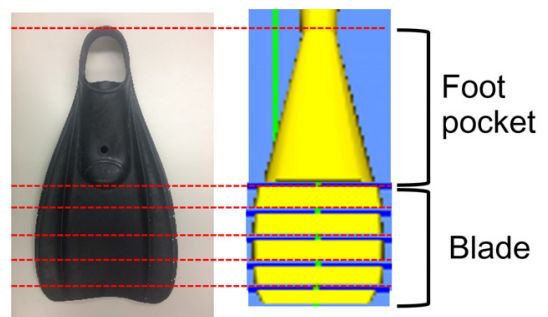

(a)

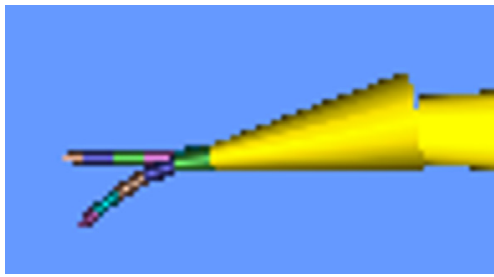

(b)

Fig. 2 The fin model in the present study. (a) The left photograph is an actual fin (NAJADE SPRINT). The right is the fin model. In the model, the foot pocket part was reproduced by modification of the geometry of the foot of the swimmer. The blade part was reproduced with five rigid plates. The geometry of each part was measured and put into the model. (b) Screenshot of the simulation reproducing the experimental situation of the bending test. Both fins with/without bending are simultaneously shown in this figure.

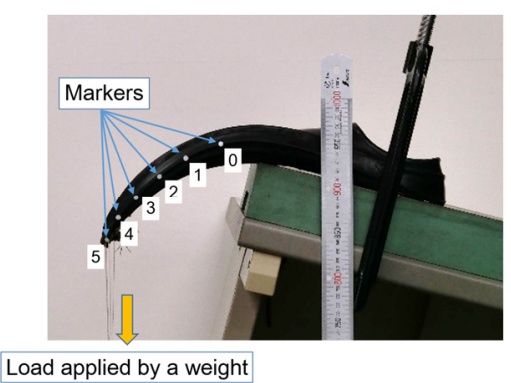

(a)

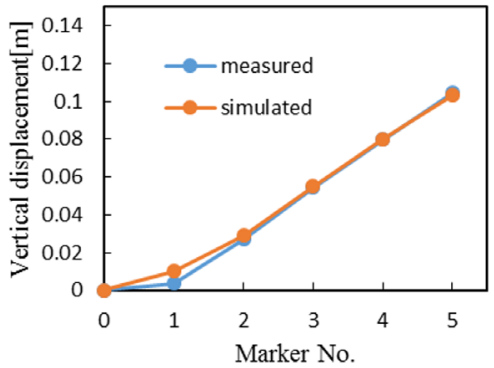

(b)

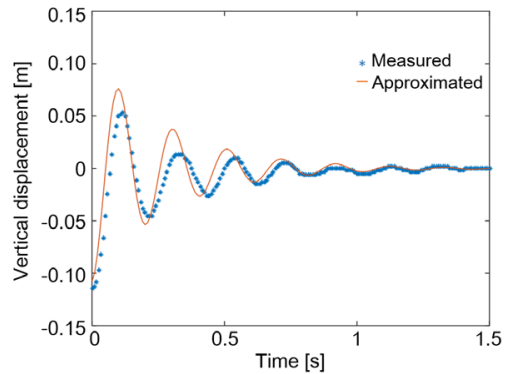

(c)

Fig. 3 Experimental setup and results for the fin bending test. (a) Experimental setup. Six markers were attached on the side edge of a fin, and a load was applied by hanging a weight at the end edge of the fin. The weight was $3 \mathrm{~kg}$ in the case of this photograph. (b) An example of experimental and simulated results of vertical displacements for the markers. A load of $5 \mathrm{~kg}$ was applied in the down kick direction in this case. (c) Experimental result of time history of vertical displacement for the edge of the fin in the dynamic bending test, in which a load of $5 \mathrm{~kg}$ was applied in the up kick direction. The results were approximated as a curve of one degree-of-freedom vibration system. 
released from the load suddenly. The time history of the vertical displacement of the edge of the fin (marker No. 5) was measured. The experimental result is shown in Fig. 3(c). The measured time history was approximated by a damping curve of one degree-of-freedom vibration system, as shown in Fig. 3(c). On the other hand, this experimental situation was again reproduced by the simulation. From the simulated vertical displacement of the edge of the fin, another curve approximated as a one-degree-of-freedom vibration system could be drawn. And the damping coefficients of the fin were determined so that those two curves were consistent with each other.

In order to determine the fluid force coefficients for the fin, an experiment was conducted. In this experiment, the fin was located vertically in a pool and was moved horizontally by an air cylinder, as shown in Fig. 4. The forces acting on the fin vertically ( $X$ axis, propulsive direction in swimming) and horizontally ( $Z$ axis, vertical direction in swimming) were measured by two force gauges, respectively. The horizontal movement of the fin was measured by a laser displacement meter. The experimental situation was reproduced by the simulation as well. The measured movement of the fin was put into the simulation, and the fluid forces acting on the fin were calculated. Note that the horizontal inertia force of the fin itself was deducted from the measured value in order to extract the fluid force only. The fluid force coefficients were manually adjusted and determined so that the experimental and simulated forces were consistent with each other. The results of the experimental and simulated fluid forces are shown in Fig. 5. It was found that the trends of the experimental results were reproduced by the simulation, although some discrepancies were found, especially for $X$ axis in the up kick motion.

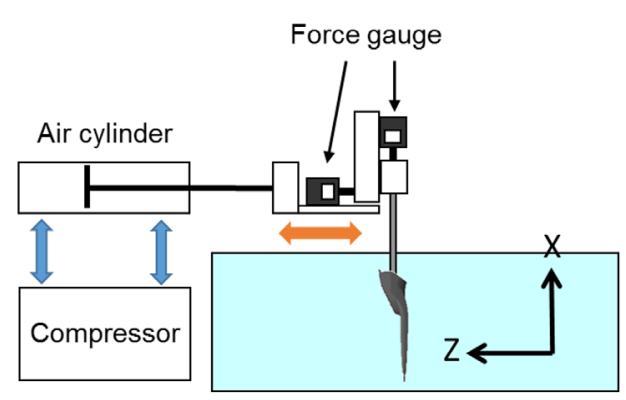

(a)

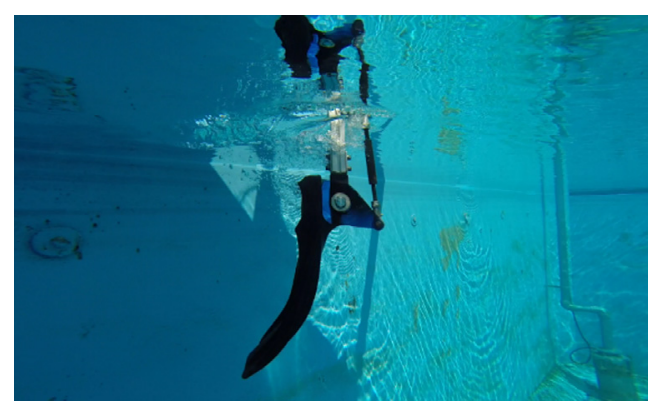

(b)

Fig. 4 Experiment to measure the fluid forces acting on a fin. (a) Schematic figure of the experimental setup. (b) Underwater view of the fin, which moved from the left to the right in this photograph.

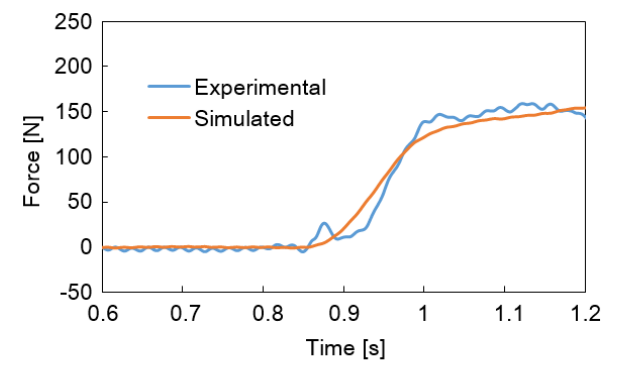

(a)

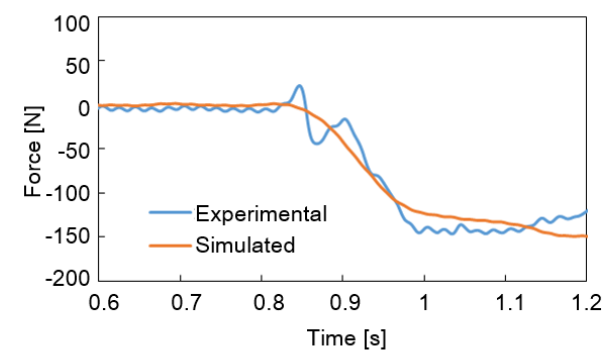

(c)

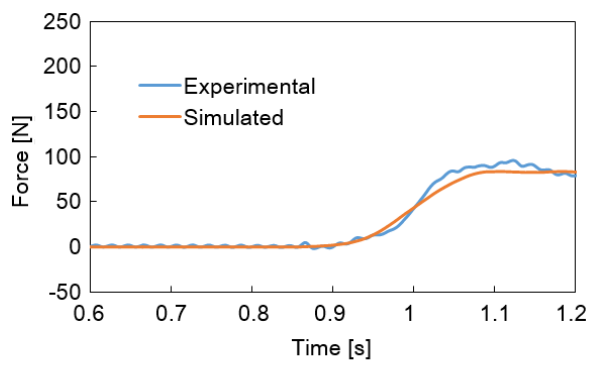

(b)

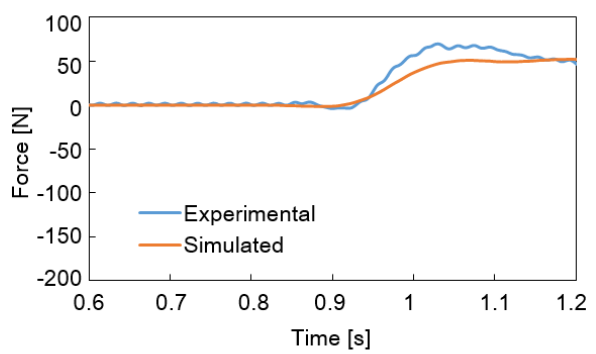

(d)

Fig. 5 Experimental and simulated fluid forces acting on a fin. (a) $Z$ axis component for down kick (b) $X$ axis component for down kick (c) $Z$ axis component for up kick (d) $X$ axis component for up kick. 


\subsection{Experiment to acquire swimming motion}

In order to acquire the motion of swimming with fins, an experiment was conducted. In this experiment, a swimmer performed maximal front crawl swimming with/without fins in an indoor swimming pool. The swimming motion was filmed at 60 frames per second by three cameras underwater (Coolpix AW110, Nikon Corp., Japan) and one camera on land (iPhone 7, Apple Inc., USA), as shown in Fig. 6. The videos taken by the four cameras were synchronized by simultaneously filming a flare from a flashlight at the beginning of the trial.

Two male collegiate bi-fin swimmers participated this study. The experimental protocol was sufficiently explained to the participants before the experiment and written consents from the participants were obtained. The experimental protocol was approved by the Ethical Committee of Tokyo Institute of Technology. Sixteen self-luminous markers were attached on the right and left side of each participant's body at eight anatomical landmarks, as shown in Fig. 7. The landmarks were the acromin, lateral epicondyle of the humerus, styloid process of the ulna, fifth metacarpal bone, greater trochanter, lateral condyle of the femur, lateral malleolus and fifth metatarsal bone. After a sufficient warming-up session, the participants were asked to swim with the NAJADE SPRINT fins and a snorkel (fin swimming trial) as well as without fins and the snorkel (free swimming trial) for $25 \mathrm{~m}$ at their maximal effort.

From the filmed video images, the stroke cycles and swimming speeds of the all of the trials were calculated. The results are shown in Table 1. It was found that the swimming speeds of fin swimming trials were faster than those of the freestyle trials. The swimming motion was also obtained from the filmed video images. The method is schematically shown in Fig. 8. To begin with, a swimmer segmental model was constructed on a three-dimensional computer graphics

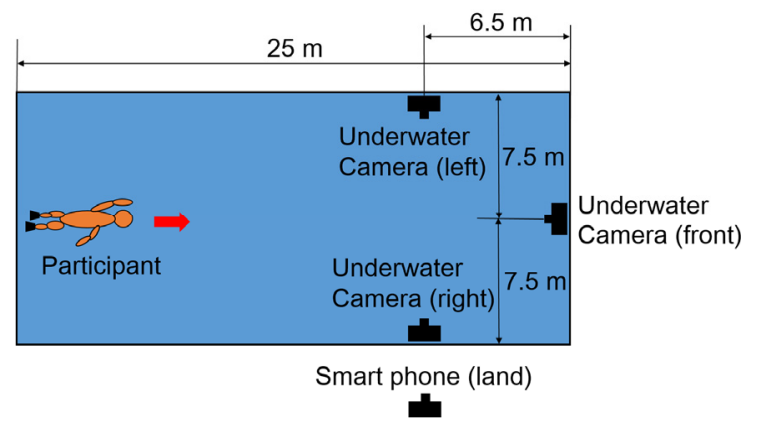

Fig. 6 Experimental setup to acquire swimming motion. The participant swam with/without fins for $25 \mathrm{~m}$ long at maximal effort. The swimming motion was filmed by three underwater and one on-land cameras.

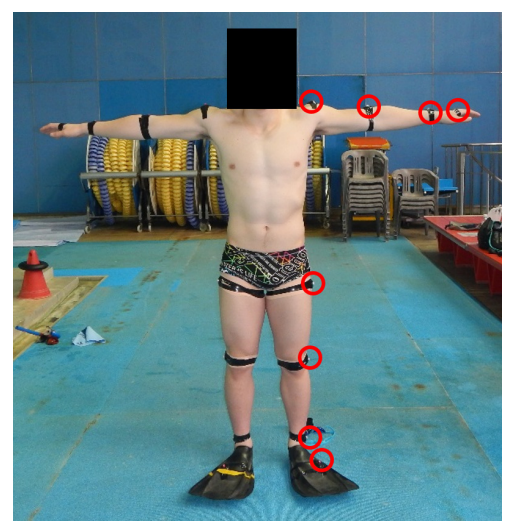

Fig. 7 Self luminous markers attached to the participant. The circles indicate the locations of the eight markers for the left side of the participant's body. Eight markers were also attached to the right side of the body.

Table 1 Stroke cycles and swimming speeds in the experiment.

\begin{tabular}{ccccc}
\hline \multirow{2}{*}{ Participant ID } & \multicolumn{2}{c}{ Fin swimming } & \multicolumn{2}{c}{ Free swimming } \\
\cline { 2 - 5 } & Stroke cycle & Swimming speed & Stroke cycle & Swimming speed \\
\hline 1 & $1.12 \mathrm{~s}$ & $1.91 \mathrm{~m} / \mathrm{s}$ & $1.10 \mathrm{~s}$ & $1.75 \mathrm{~m} / \mathrm{s}$ \\
2 & $1.10 \mathrm{~s}$ & $1.85 \mathrm{~m} / \mathrm{s}$ & $1.13 \mathrm{~s}$ & $1.50 \mathrm{~m} / \mathrm{s}$ \\
\hline
\end{tabular}




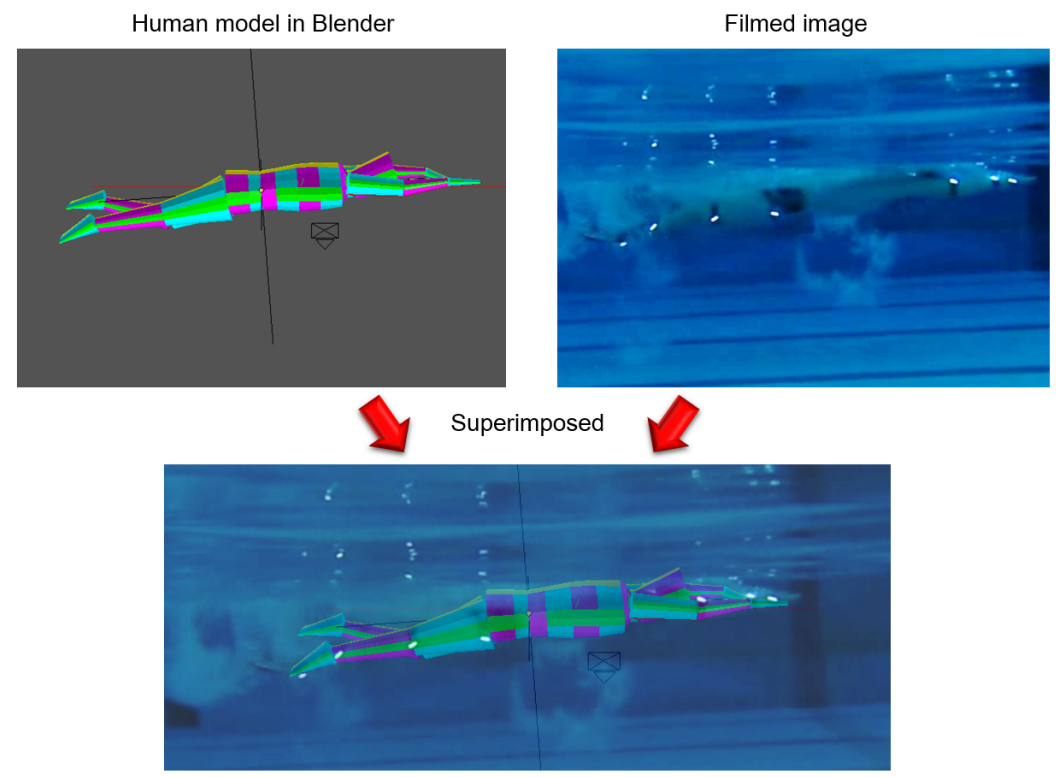

Fig. 8 Swimming motion as joint angles were determined by superimposing the image of the human model with the filmed image on Blender.

software Blender (Blender Foundation, The Netherlands), as shown in the left top of Fig. 8. This model had the same degrees-of-freedom as those in SWUM, and the sizes of the segments were adjusted for each participant based on the photographs of the participant, which were taken just before the swimming experiment. The images of the model were then superimposed on the video images of the experiment, as shown in the bottom of Fig. 8. On each superimposed image, the joint angles of the model were adjusted by the software and determined so that the model posture became consistent with the filmed posture as much as possible.

\section{Results and Discussion}

\subsection{Validation by comparison with experimental results}

Using all the input data described in the previous chapter, simulations reproducing the experimental trials were carried out. Five stroke cycles were calculated for each trial to eliminate the effect of initial conditions. As an example, the animation images of simulated movement for the fin swimming of participant 1 are shown in Fig. 9. The symbol $t^{*}$ is the nondimensional time normalized by the stroke cycle. Thus, the duration of $t^{*}=4.0 \sim 5.0$ corresponds to the last stroke

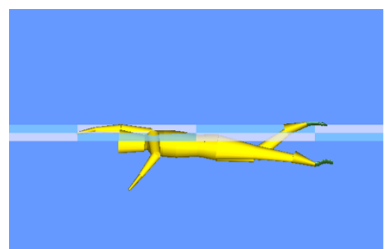

(a) $t^{*}=4.000$

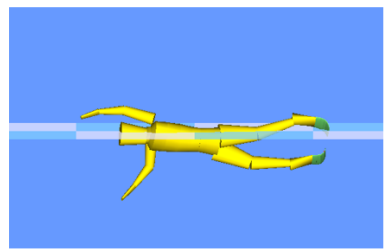

(e) $t^{*}=4.500$

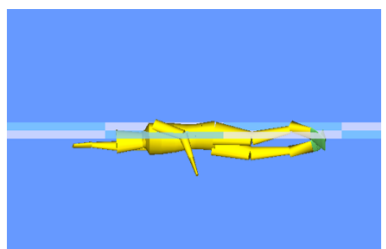

(b) $t^{*}=4.125$

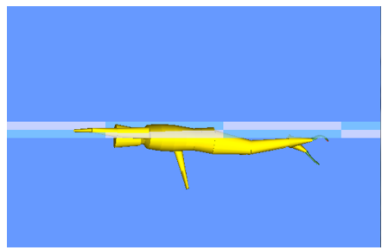

(f) $t^{*}=4.675$

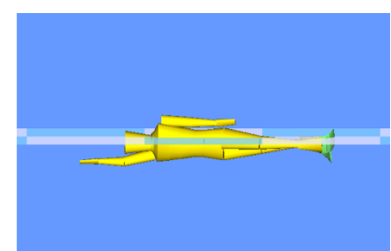

(c) $t^{*}=4.250$

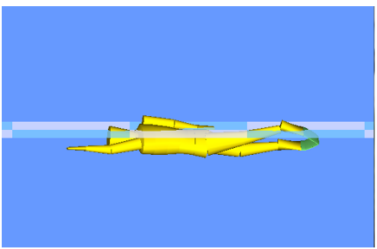

(g) $t^{*}=4.750$

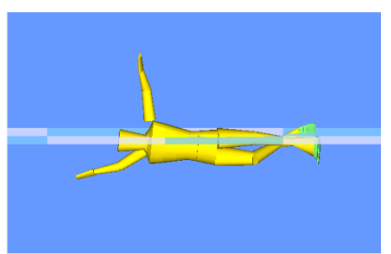

(d) $t^{*}=4.375$

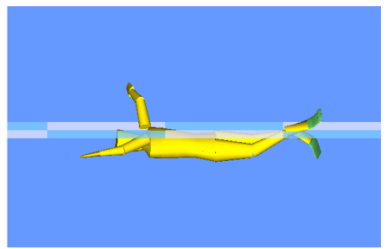

(h) $t^{*}=4.875$

Fig. 9 Animation images of simulated movement for fin swimming of participant 1 . The symbol $t^{*}$ is the nondimensional time normalized by the stroke cycle. Therefore, the duration of $t^{*}=4.0 \sim 5.0$ corresponds to the final stroke cycle. 
Table 2 Swimming speeds in the simulation and experiment.

\begin{tabular}{ccccc}
\hline Participant ID & Trial & Experiment [m/s] & Simulation [m/s] & Discrepancy [\%] \\
\hline \multirow{2}{*}{1} & Fin & 1.91 & 1.98 & 3.66 \\
& Free & 1.75 & 1.72 & -1.71 \\
\hline \multirow{2}{*}{2} & Fin & 1.85 & 1.92 & 3.78 \\
& Free & 1.50 & 1.42 & -5.33 \\
\hline
\end{tabular}

cycle. The blades of the fins are colored in green. It was confirmed that the blades bent due to the fluid forces. For the validation of the simulation, the swimming speeds of all the trials are shown in Table 2. In the experiment, the swimming speeds of the fin swimming were significantly faster than those of the free swimming with both participants. The swimming speeds in the simulation exhibited the same tendency. In addition, the quantitative discrepancies of the simulated values from the experimental ones were calculated, as shown in the rightmost column in Table 2. It was found that all discrepancies were within $6 \%$. This suggests the validity of the present simulation method.

\subsection{Parameter study with respect to amplitude of ankle joints}

Using the validated simulation method, a parameter study with respect to the amplitude of ankle joints was conducted. In this parameter study, "amplitude ratio" was defined. For example, if the amplitude ratio was 0.5 , all the original angles at the ankle joints were multiplied by 0.5 . The amplitude ratios were changed to $0.75,0.5,0.25$ and 0 both for fin swimming and free swimming. The results are shown in Fig. 10. It was found that swimming speeds of fin swimming increased as the amplitudes decreased for both participants, while the swimming speeds for free swimming decreased as the amplitudes decreased. In other words, swimming with fixed ankle joints brought higher swimming speed than that with moving ankle joints in fin swimming. In order to discuss the reason for this tendency, animation images of the foot and fin in the simulation at the same moment during down kick are shown in Fig. 11. This figure is to show the bending magnitude of the elastic blade part of the fin. The red circles are put in the figure to show the elastic blade part

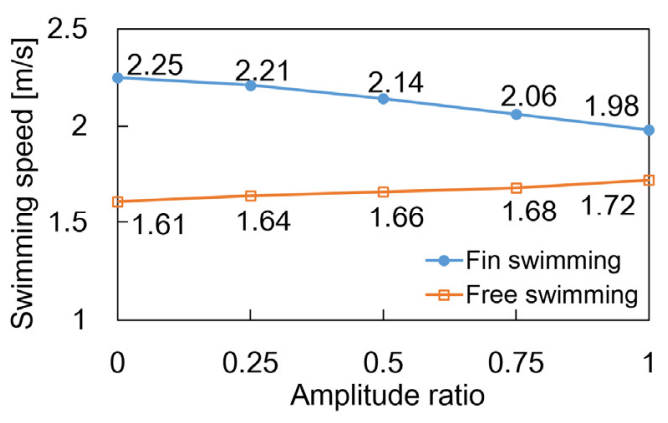

(a) Participant 1

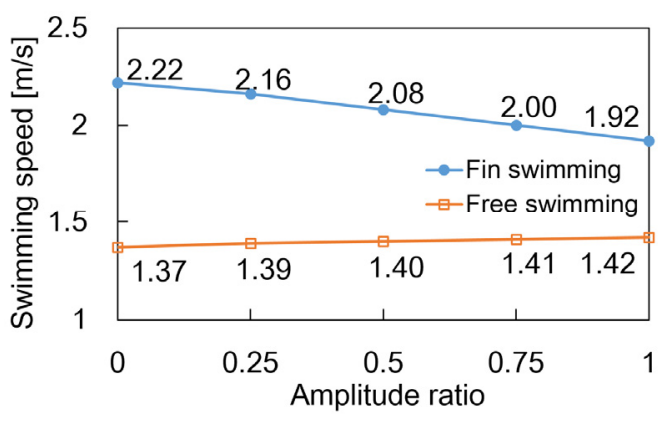

(b) Participant 2

Fig. 10 Relationship between amplitude of joint angle at ankle and swimming speed.

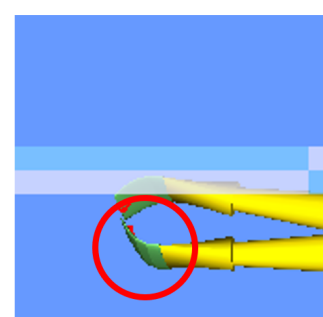

(a)

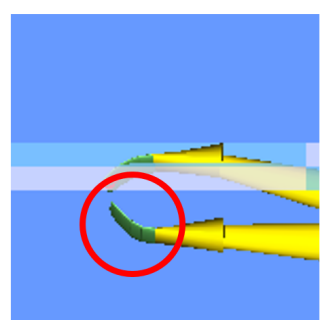

(b)

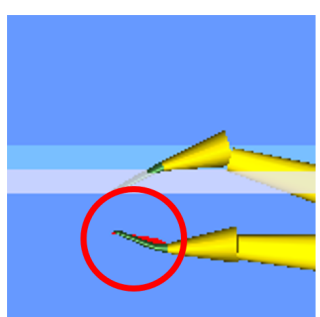

(c)

Fig. 11 Animation images of the foot and fin in the simulation at the same moment during down kick (Participant 1). a Amplitude was 0 (fixed ankle joints). b Amplitude was 0.5 times. c Amplitude was 1.0 times (original). This figure is to show the bending magnitude of the elastic blade part of the fin. The red circles are put in the figure to show the elastic blade part of the fin (green part). 
of the fin (green part). As shown in this figure, it was found that the fin was bent due to the fluid force when the amplitude was zero (fixed ankle joints) most. As a result, the thrust produced by the fin became larger when the amplitude was zero. On the other hand, the bending of the fin was small when the amplitude was 1.0 times (original), resulting in a smaller thrust. Therefore it can be concluded that the fin swimmer should not move the ankle joints and maintain a constant angle to improve performance. The ankle joint movements in the original fin swimming trial by the participants might be induced by excessive fluid force acting on the fin, and the ankle joints could not support such a large force.

\subsection{Discussion about ankle joint torque}

An example of ankle joint torque calculated in the simulation is shown in Fig. 12 (the right ankle of the participant 1). From this figure, it was found that the positive (dorsi-flexing) torque was exerted by the swimmer in the down kicks, while the negative (plantar-flexing) torque was exerted by the swimmer in the up kicks, although the curves had many high-frequency fluctuations due to the connection by the virtual springs and dampers. The comprehensive results of the ankle joint torque in the simulation are shown in Table 3. From this table, it was found that the ankle joint torque increased both in the dorsi-flexing and plantar-flexing directions. It was also found that the joint torque itself as well as the increase in magnitude of the participant 1 was larger than those of the participant 2 . It may be due to the difference in the kicking

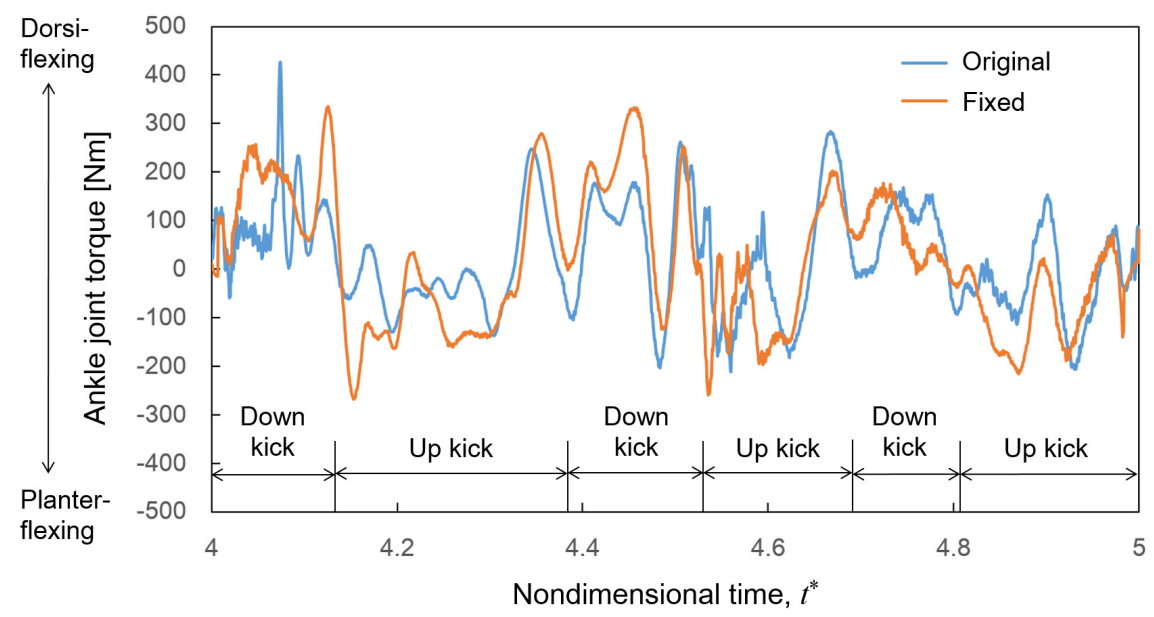

Fig. 12 An example of ankle joint torque calculated in the simulation (the right ankle of the participant 1). The results for the original ankle joint amplitude as well as those for the fixed case (the amplitude was zero) are shown. The positive ankle joint torque means the torque was exerted by the swimmer in the dorsi-flexing direction, while the negative means that the torque was exerted in the plantar-flexing direction. In one stroke cycle $\left(t^{*}=4.0 \sim 5.0\right)$, three up kicks and three down kicks were performed, respectively.

Table 3 Averaged ankle joint torques calculated in the simulation. In the table, for example, the first value of $56.0 \mathrm{Nm}$ was calculated by taking an average of all dorsi-flexing time regions for the right ankle of the participant 1 (the average of all time regions in which the joint torque became positive in Fig. 12). The increase in magnitude was calculated by dividing the joint torque of the fixed case by that of the original, and taking the average of the right and left.

\begin{tabular}{|c|c|c|c|c|c|}
\hline & Direction & $\begin{array}{l}\text { Right(R) or } \\
\text { Left(L) }\end{array}$ & Original [Nm] & Fixed [Nm] & $\begin{array}{l}\text { Increase in } \\
\text { magnitude }\end{array}$ \\
\hline \multirow{4}{*}{ Participant 1} & \multirow{2}{*}{ Dorsi-flexing } & $\mathrm{R}$ & 56.0 & 66.8 & \multirow{2}{*}{$25.6 \%$} \\
\hline & & $\mathrm{L}$ & 64.6 & 85.2 & \\
\hline & \multirow{2}{*}{ Plantar-flexing } & $\mathrm{R}$ & -33.5 & -50.4 & \multirow{2}{*}{$42.7 \%$} \\
\hline & & $L$ & -36.4 & -49.2 & \\
\hline \multirow{4}{*}{ Participant 2} & \multirow{2}{*}{ Dorsi-flexing } & $\mathrm{R}$ & 30.6 & 28.6 & \multirow{2}{*}{$2.1 \%$} \\
\hline & & $\mathrm{L}$ & 41.0 & 45.5 & \\
\hline & \multirow{2}{*}{ Plantar-flexing } & $\mathrm{R}$ & -28.6 & -29.0 & \multirow{2}{*}{$4.5 \%$} \\
\hline & & $\mathrm{L}$ & -22.8 & -24.5 & \\
\hline
\end{tabular}


style of the participants. The kicking style of the participant 1 might be regarded as more powerful one, while that of the participant 2 might be regarded as more efficient one. With respect to the magnitude of the joint torque, the average maximum voluntary ankle joint torques for ordinary 20 to 29 years old males were reported as $40.7 \mathrm{Nm}$ for dorsi-flexion and 126.5 Nm for plantar-flexion, respectively (Moraux et al., 2013). Although well-trained athletes might be able to exert larger joint torque, the fin swimmers have to switch the direction of the joint torque very frequently according to the kicking motion. Therefore, from the results of the present study, it is difficult to conclude whether the fixed ankle in the present simulation is really possible or not by an actual swimmer. However, it probably can be said that the dorsiflexing torque in the down kick is more likely to be insufficient than the planter flexing torque in the up kick, since the maximum dorsi-flexing torque is much smaller than the maximum plantar-flexing torque. If the joint torque is insufficient, the foot is forcibly moved by the external fluid forces. In that case, the joint angle reached the limit and therefore the extra torque will be supported by tendons and ligaments of the joints. This might be a cause of injury. Therefore, it is important for the bi-fin swimmers to train the ankle joint especially for the dorsi-flexing direction not only to improve the performance, but also to prevent injury.

\subsection{Parameter study with respect to fixed angle of ankle joints}

As described above, it was found that the ankle joints should be fixed at a constant angle to improve performance. Therefore, a parameter study with respect to the fixed angle was conducted next. In this parameter study, the ankle joints were fixed at a constant angle, which was changed as a parameter. The results are shown in Fig. 13. A positive fixed angle of the ankle joints means the dorsi-flexed direction while a negative angle means the plantar-flexed direction. When the angle is zero, the foot segment becomes parallel to the shank segment. It was found that the maximal swimming speed occurred when the angle was zero degrees for the participant 1, and when the angle was -10 degrees for participant 2. These results suggested that there were optimal fixed angles of ankle joints to maximize swimming speed. In order to examine the results in more detail, the body pitch angle, which was defined as the angle of the lower waist segment in the pitch direction averaged for one stroke, was calculated. When the lower waist segment is horizontal, the body pitch angle is zero degrees. The results are shown in Fig. 14 together with the animation snapshots and swimming speeds. From this figure, it was found that the body pitch angle became larger (more upright) when the fixed angle of ankle joints was smaller (more plantar-flexed) for both of the participants. This was because the kick with more plantar-flexed position produced more downward-pointing fluid forces, resulting in sinking the lower body of the swimmer. As a result, it induces a decrease in the swimming speed since the projected area of the swimmer's body became large. On the other hand, when the fixed angle of ankle joints was smaller (more dorsi-flexed), the body pitch angle became smaller (more horizontal). However, an excessively horizontal body position induces kicks above the water surface, which also resulted in a decrease in the swimming speed. Therefore, it can be concluded that there is an optimum fixed angle of ankle joints for a swimmer to maximize the swimming speed.

\section{Conclusion}

In the present study, fin swimming using bi-fins was analyzed by the developed simulation method. The developed

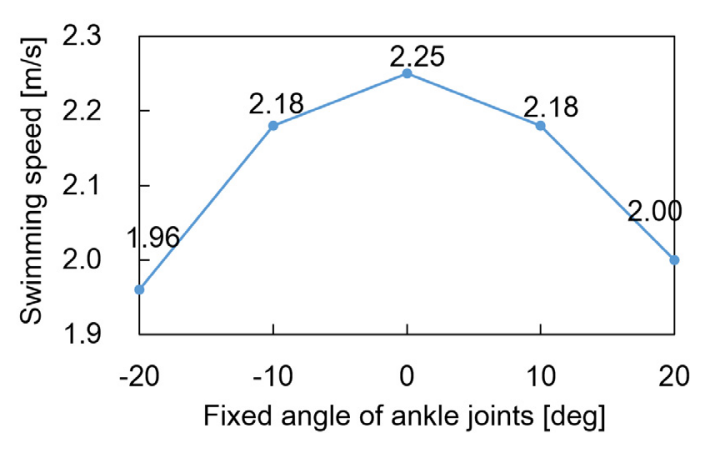

(a) Participant 1

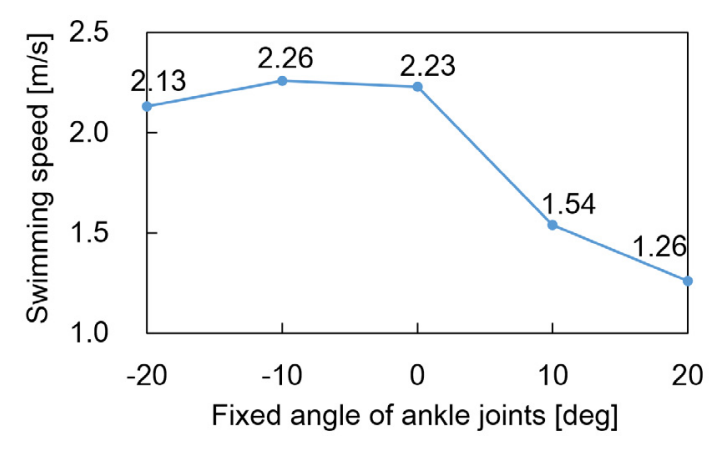

(b) Participant 2

Fig. 13 Relationship between fixed angle of ankle joints and swimming speed. 


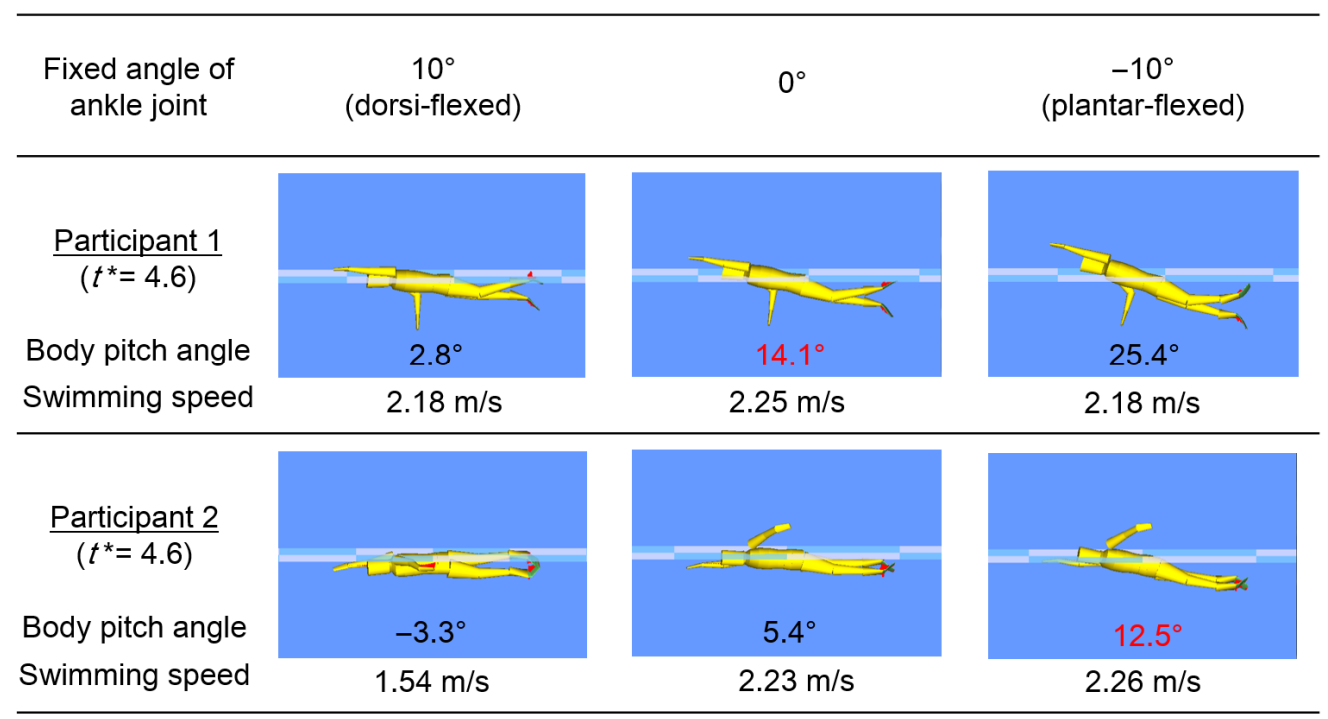

Fig. 14 Relationship between the fixed angle of ankle joints and body pitch angle. Animation snapshots at $t^{*}=4.6$ for the two participants are shown for comparison. Body pitch angle and swimming speed averaged for one stroke are shown at the bottom of each image.

simulation method was validated since the simulated swimming speeds were consistent with the experimental values in errors less than $6 \%$ of accuracy. By analyses with the validated simulation method, it was found that the smaller ankle joint movements resulted in a faster swimming speed. It was also found that the fixed angle of ankle joints affected the body pitch angle and that there is an optimum fixed angle for a swimmer to maximize swimming speed. The developed simulation method can be applied to any other bi-fins and swimmers by changing the input parameters. The method is promising to become a useful tool for investigating the mechanics of fin swimming.

\section{References}

Bideau, N., Razafimahery, F., Monier, L., Mahiou, B., Nicolas, G., Bideau, B. and Rakotomanana, L., 3D Computational Fluid-structure Interaction Model for the Estimation of Propulsive Forces of a Flexible Monofin, Biomechanics and Medicine in Swimming XI, (2010), pp.52-54.

Kiuchi, H., Nakashima, M., Cheng, K.B. and Hubbard, M., Modeling fluid forces in the dive start of competitive swimming, Journal of Biomechanical Science and Engineering, Vol.5, (2010), pp.314-328.

Luersen, M.A., Le Riche, R., Lemosse, D. and Le Maitre, O., A Computationally Efficient Approach to Swimming Monofin Optimization, it Structural and Multidisciplinary Optimization, Vol.31, (2006), pp.488-496.

Moraux, A., Canal, A., Ollivier, G., Ledoux, I., Doppler, V., Payan, C. and Hogrel, J., Ankle dorsi- and plantar-flexion torques measured by dynamometry in healthy subjects from 5 to 80 years, BMC Musculoskeletal Disorders, Vol. 14, (2013), p.104.

Nakashima, M., Mechanical study of standard six beat front crawl swimming by using swimming human simulation model, Journal of Fluid Science and Technology, Vol.2, (2007), pp.290-301.

Nakashima, M., Miura, Y. and Satou, K., Development of swimming human simulation model considering rigid body dynamics and unsteady fluid force for whole body, Journal of Fluid Science and Technology, Vol.2, (2007), pp.5667.

Nakashima, M., Simulation analysis of the effect of trunk undulation on swimming performance in underwater dolphin kick of human, Journal of Biomechanical Science and Engineering, Vol.4, (2009), pp.94-104.

Nakashima, M., Modeling and simulation of human swimming, Journal of Aero Aqua Bio-mechanisms, Vol.1, (2010), pp.11-17.

Nakashima, M., Kiuchi, H. and Nakajima, K., Multi agent/object simulation in human swimming, Journal of Biomechanical Science and Engineering, Vol.5, (2010a), pp.380-387.

Nakashima, M., Suzuki, S. and Nakajima, K., Development of a simulation model for monofin swimming, Journal of 
Biomechanical Science and Engineering, Vol.5, (2010b), pp.408-420.

Nakashima, M., Maeda, S., Miwa, T. and Ichikawa, H., Optimizing simulation of the arm stroke in crawl swimming considering muscle strength characteristics of athlete swimmers, Journal of Biomechanical Science and Engineering, Vol.7, No.2 (2012), pp.102-117.

Nakashima, M., Suzuki, S., Ono A. and Nakamura, T., Development of the transfemoral prosthesis for swimming focused on ankle joint motion, Journal of Biomechanical Science and Engineering, Vol.8, (2013), pp.79-93.

Nakashima, M. and Ono, A., Maximum joint torque dependency of the crawl swimming with optimized arm stroke, Journal of Biomechanical Science and Engineering, Vol.9, No.1, (2014), pp.1-9.

Nakashima, M., Nemoto, C., Kishimoto, T., Terada, M. and Ikuta, Y., Optimizing simulation of arm stroke in freestyle for swimmers with hemiplegia, Mechanical Engineering Journal, Vol.5, (2018), Pages 17-00377.

Nicolas, G., Bideau, B., Bideau, N., Colobert, B., Guerroue, G.L. and Delamarche, P., A new system for analyzing swim fin propulsion based on human kinematic data, Journal of Biomechanics, Vol.43, (2010), pp.1884-1889.

Ongun, M.K., Frequency in Freestyle Swimming, World Applied Sciences Journal, Vol.30, (2014), pp.1070-1074.

Zamparo, P., Pendergast, D.R., Termin, B. and Minetti, A.E., How fins affect the economy and efficiency of human swimming, The Journal of Experimental Biology, Vol.205, (2002), pp.2665-2676.

Zamparo, P., Pendergast, D.R., Termin, B. and Minetti, A.E., Economy and efficiency of swimming at the surface with fins of different size and stiffness, European Journal of Applied Physiology, Vol.96, (2006), pp.459-470. 